\title{
ESTABLISHMENT OF STABLE CELL LINE FOR INDUCING KAP1 PROTEIN EXPRESSION
}

\author{
XiaOyan Liu, ** Md. AsaduzZaman Khan, ** Jingliang ChEng, \\ Chunli Wei, ${ }^{* *}$ Lianmei Zhang and JunJiang Fu**** \\ Key Laboratory of Epigenetics and Oncology, The Research Center for Preclinical Medicine, \\ Luzhou Medical College, 3-319, Zhongshan road, Luzhou, Sichuan 646000, P. R. China
}

(Received: June 5, 2014; accepted: August 6, 2014)

\begin{abstract}
Generation of the stable cell lines is a highly efficient tool in functional studies of certain genes or proteins, where the particular genes or proteins are inducibly expressed. The KRAB-associated protein-1 (KAP1) is an important transcription regulatory protein, which is investigated in several molecular biological studies. In this study, we have aimed to generate a stable cell line for inducing KAP1 expression. The recombinant plasmid pcDNA5/FRT/TO-KAP1 was constructed at first, which was then transfected into Flp-InTMT-REx TM-HEK293 cells to establish an inducible pcDNA5/FRT/TO-KAP1-HEK293 cell line. The Western blot analysis showed that the protein level of KAP1 is over-expressed in the established stable cell line by doxycycline induction, both dose and time dependently. Thus we have successfully established stable pcDNA5/FRT/TO-KAP1-HEK293 cell line, which can express KAP1 inducibly. This inducible cell line might be very useful for KAP1 functional studies.
\end{abstract}

Keywords: KRAB-associated protein-1 - protein expression - recombinant plasmid transfection - inducible cell line

\section{INTRODUCTION}

Stable cell lines are important in molecular cell biology research, in which one or more genes/proteins are activated or over-expressed by genetic engineering. In functional studies of proteins/protein complexes, efficient and stable cell lines for overexpression of particular proteins are essential. Generation of inducible stable cell lines thus performs as a highly efficient tool in functional, molecular and cellular biology research. The KRAB-associated protein-1 (KAP1), also known as Tripartite motif-containing 28 (TRIM28) or transcriptional intermediary factor $1 \beta$ (TIF1 $\beta$ ) is a mammalian protein that is involved in the regulation of transcription process [12, 13]. KAP1 is involved in multiple cellular processes, including gene silencing, cell growth and differentiation, pluripotency, neoplastic transformation, apoptosis, DNA repair, and maintenance of genomic integrity in mammalian cells [8]. KAP1 is also supposed to play an important role in ubiquitination process because of its interaction with E3

\footnotetext{
* Corresponding author; e-mail address: fujunjiang@hotmail.com

**Equal contribution.
} 
ligase protein MDM2, which is involved in several functional regulations [15]. Furthermore, this protein has been reported to be up-regulated in different cancers, and implicated in the activation or repression of transcriptional process of several genes [9]. For example, upregulated KAP1 provides a survival advantage to gastric and lung cancer cells, and is speculated as a potential diagnostic marker of cancer metastasis $[11,16]$.

Although KAP1 is involved in a number of cellular processes, the appropriate role of this protein is not clearly understood. To further investigate the function of KAP1, a stable cell line, which can expresses KAP1 inducibly, would be beneficial in related research fields. In this study, we have established a stable cell line (pcDNA5/FRT/ TO-KAP1-HEK293), which can express KAP1 inducibly by the addition of doxycyclin (DOX) after cloning the KAP1 ORF into modified pcDNA5/FRT/TO vector. We have characterized the established cell's KAP1 expression with DOX induction by Western blot analysis.

\section{MATERIALS AND METHODS}

\section{Chemicals}

For cell culture, Dulbecco's Modified Eagle's medium (DMEM) and Fetal Bovine Serum (FBS) were purchased from Life Technologies (USA). The vector pcDNA5/ FRT/TO was purchased from Invitrogen (USA) and modified, in which the FLAG was tagged. Flp-IntMT-REx ${ }^{\mathrm{TM}}-293$ cell line and pOG44 plasmids were purchased from Invitrogen (USA), FLAG and Tubulin antibodies were purchased from Sigma in USA, and pcDNA3-KAP1 was kindly given by Dr. Jiemin Wong from Baylor College of Medicine (Houston, TX, USA).

\section{Construction of pcDNA5/FRT/TO-KAP1 recombinant plasmid}

With the pcDNA3-KAP1 plasmid as a template, PCR fidelity polymerase was performed to amplify full length ORF KAP1. The amplification primers were: KAP15EcoRV: 5'-GTGGATATCGCGGCCTCCGCGGCGG-3', KAP1-3NheI: 5'-GCTGCTAGCGGGGCCATCACCAGGGC-3'. The expression vector pcDNA5/ FRT/TO was double digested with $E c o R V$ and NheI restriction enzymes, and with the same endonuclease, KAP1 was digested and then ligated. Positive clones were verified by restriction enzyme treatment, PCR amplification and DNA sequencing.

\section{Cell transfection}

Flp-In ${ }^{\mathrm{TMT}}$-REx ${ }^{\mathrm{TM}}-293$ cells were cultured in complete medium containing DMEM, $10 \%$ FBS in a 6 -well culture plate. When the cultured cell density reached at about 
$40 \%$, the medium was aspirated, and washed twice with phosphate buffered saline $(1 \times$ PBS $)$. The recombinant pcDNA5/FRT/TO-KAP1 $(1.8 \mu \mathrm{g})$ and pOG44 $(0.2 \mu \mathrm{g})$ were co-transfected, and as control, pOG44 was co-transfected with pcDNA5/FRT/ TO empty vector [3].

\section{Screening of positive clones}

Stable transfectants were screened using hygromycin B (Invitrogen) treatment. Transfected cells were treated with hygromycin B $(200 \mu \mathrm{g} / \mathrm{ml})$, and after 5-10 days, KAP1 stable transfected cell lines were screened out successfully (hygromycin B killed the negative cells).

\section{Measurement of the effect of different doses of doxycycline on induced cell line expressing KAP1}

The stable pcDNA5/FRT/TO-KAP1-HEK293 cells were cultured in duplicate in a 12 well plate with the treatment of $0,0.001 \mu \mathrm{g} / \mathrm{ml}, 0.01 \mu \mathrm{g} / \mathrm{ml}, 0.1 \mu \mathrm{g} / \mathrm{ml}, 0.5 \mu \mathrm{g} / \mathrm{ml}$ and $1 \mu \mathrm{g} / \mathrm{ml}$ DOX. After 16 hours (h) treatment, the culture medium was aspirated; the cells were washed with $1 \times \mathrm{PBS}$ twice, and collected in $1.5 \mathrm{ml}$ centrifuge tubes. Each group of cells was treated with $120 \mu \mathrm{EBC}$ lysis buffer for $15 \mathrm{~min}$ on ice $[4,5]$, and then centrifuged for 15 minutes $(12,000 \times \mathrm{g})$ to prepare KAP1 protein solution. The protein solution was then transferred into a new tube and mixed with equal volume of SDS lysis buffer. The mixture was heated in boiling water for $5 \mathrm{~min}$, and used for Western blotting analysis.

\section{Measurement of the effect of different times of DOX treatment on induced cell lines expressing KAP1}

With the same dose of DOX $(0.1 \mu \mathrm{g} / \mathrm{ml})$, pcDNA5/FRT/TO-KAP1-HEK293 cells were treated for $0,1,2,4,8$ and 16 hours, and cells were harvested and protein samples were prepared as above mentioned for Western blot analysis.

\section{Western blot analysis}

The proteins in solutions were separated by SDS-PAGE (10\% separating gel and 5\% stacking gel), and then transferred onto nitrocellulose membrane. The membrane was first kept in 5\% skimmed milk (in $1 \times$ TBST) at room temperature for $2 \mathrm{~h}$ with gentle shaking. Then the membrane was incubated with primary antibody monoclonal antiFLAG (Sigma, USA) at $4{ }^{\circ} \mathrm{C}$ for $12 \mathrm{~h}$ with gentle shaking. Mouse anti-human tubulin monoclonal antibody (Sigma, USA) was used as control. The membrane was then 
washed thrice with $1 \times$ TBST, and incubated with secondary antibody tagged with horseradish peroxide [Goat Anti-Mouse IgG $(\mathrm{H}+\mathrm{L}) \mathrm{HRP}$, Bioworld] at room temperature for $2 \mathrm{~h}$. The membrane was washed thrice again with $1 \times$ TBST, and the images of protein expression were documented using the ChemiDoc XR (UNIVERSAL HOOD II-S.N, 76S/03098, Bio-Rad, USA) after the electro-chemiluminescent (ECL) reaction [3].

\section{RESULTS}

\section{Establishment of pcDNA5/FRT/TO/KAP1 vector and cell line}

With pcDNA3-KAP1 as a template, fidelity PCR polymerase amplification of fulllength KAP1 gene was performed. The expression vector pcDNA5/FRT/TO was under double digestion with EcoRV and NheI, and ligated with KAP1 was digested by the same endonuclease. Positive clones, identified by restriction enzyme digestion and PCR analysis confirmed the inserting KAP1 gene into vector (Fig. 1A). Sequencing analysis of the KAP1 sequence further verified and confirmed its correctness. Thus, recombinant pcDNA5/FRT/TO/KAP1 vector was constructed as expression vector. The vectors pcDNA5/FRT/TO/KAP1 and pOG44 were co-transfected and made pcDNA5/FRT/TO-KAP1-HEK293 (293KAP1) stable cell line. Photograph of the cell morphology is presented in Fig. 1B. The vector pcDNA5/FRT/TO and pOG44 were co-transfected and made pcDNA5/FRT/TO HEK293 cell line (293 control) (data not shown).

\section{Inducible expression of pcDNA/KAP1 from stable cell line}

KAP1 over-expression was not detected in control cell line, transfected with empty vector, where DOX was either added or not; while KAP1 cell line had a strong KAP1 expression after DOX treatment (Fig. 1C).

\section{Effects of different doses of DOX on KAPI expression in inducible cell line}

In order to evaluate the effect of DOX treatment on KAP1 protein expression in the stable cell line, we used different doses of DOX to treat pcDNA5/KAP1 cell line. Western blot analysis showed that KAP1 expression was not detected without DOX, but with DOX at $0.001 \mu \mathrm{g} / \mathrm{ml}$, KAP1 expression was detected with weak expression. With the increasing conc. of DOX (at $0.01 \mu \mathrm{g} / \mathrm{ml}$ ), KAP1 expression was increased significantly, however, KAP1 expression was not increased with further increasing the conc. of DOX, that means KAP1 was stably expressed at a certain dose of DOX (Fig. 2). 


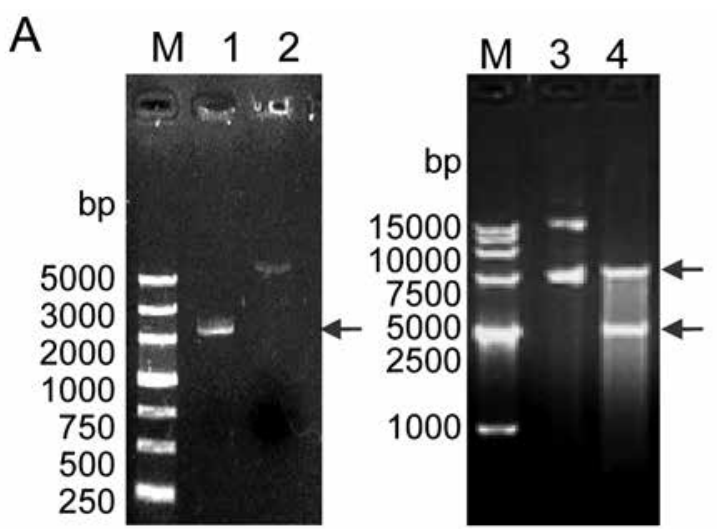

B

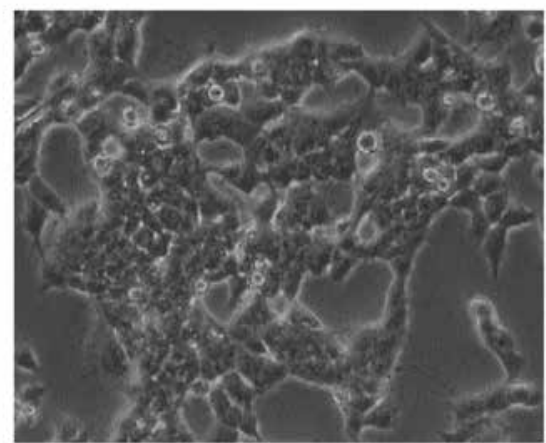

C

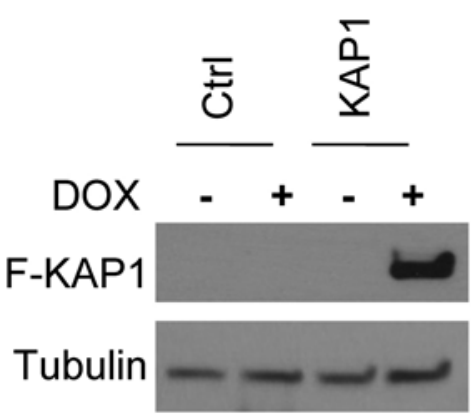

293 inducible cells

Fig. 1. KAP1 cloning and expression in inducible cell line. A) Construction of pcDNA5/FRT/TO/KAP1 recombinant plasmid. Lane "M" represents DNA marker with indicated molecular weight size $(\mathrm{kb})$; lanes 1, 2, 3 and 4 indicate KAP1 ORF, pcDNA5/FRT/TO with EcoRV and NheI digestion, recombinant pcDNA5/FRT/TO/KAP1 plasmid and recombinant pcDNA5/FRT/TO-KAP1 with EcoRV and NheI digestion respectively. B) Morphology of Flp-InTMT-REx TM-KAP1-293 cells. C) Western blot result of FLAG-KAP1. Ctrl: Flp-In ${ }^{\mathrm{TMT}}-\mathrm{REx}^{\mathrm{TM}}{ }_{-293}$ cells. KAP1: Flp-In"TMT-REx ${ }^{\mathrm{TM}}-\mathrm{K} A P 1293$ cells

\section{Effects of different duration of DOX treatment on KAP1 expression in inducible cell line}

We have used $0.1 \mu \mathrm{g} / \mathrm{ml}$ of DOX for different time duration to treat KAP1 cell line to study the time dependent DOX effect. Western blot analysis showed that KAP1 expression was not detected after $0 \mathrm{~h}$, but expressed very weakly after the treatment for $1 \mathrm{~h}$. After $2 \mathrm{~h}$, KAP1 expression was detected, which was increased gradually to the highest level after $16 \mathrm{~h}$ of treatment (Fig. 3). 
A

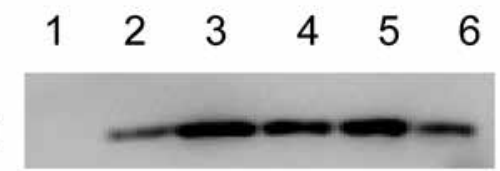

Tubulin

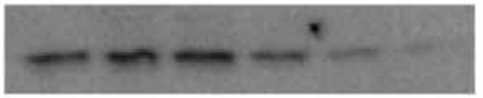

B

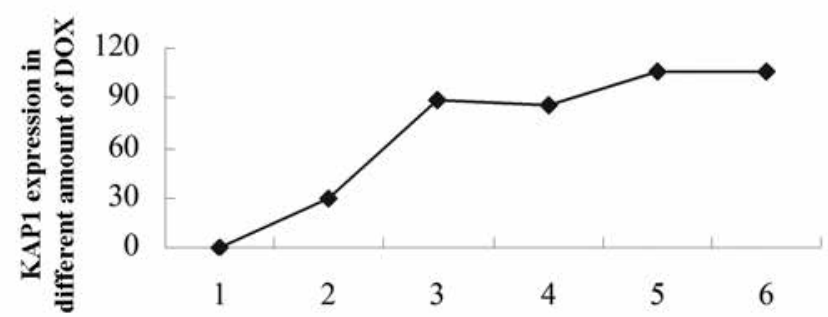

Fig. 2. Effects of different doses of DOX on KAP1 expression in inducible cell line. Lanes 1-6 indicate the amount of DOX treatment with the concentration of $0,0.001,0.01,0.1,0.5$ and $1 \mu \mathrm{g} / \mu \mathrm{l}$ (final), respectively

A

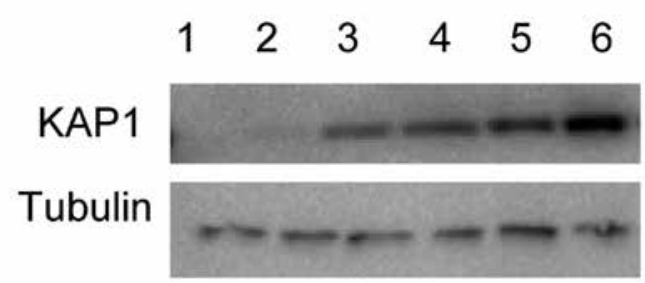

B

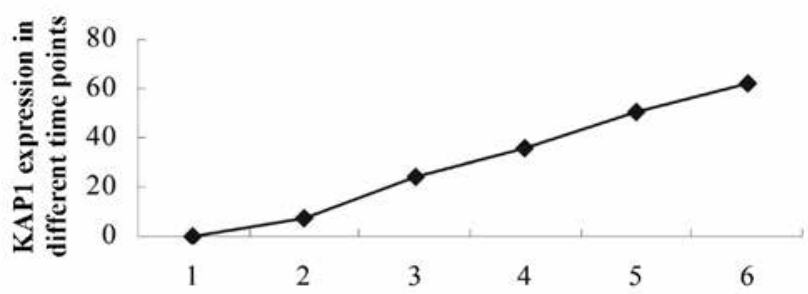

Fig. 3. Effects of different duration of DOX treatment on KAP1 expression in inducible cell line.

Lanes 1-6 indicate the treatment hours of $0,1,2,4,8$ and 16 


\section{DISCUSSION}

KAP1 is a member of tripartite motif family, which is a critical regulator of the developmental and differentiation process, especially in transcriptional step of genomic expression [8]. Abnormal expression or deletion of KAP1 has been implicated in several physiological and biochemical complications or diseases, such as, cancer development, impairment of learning and memory, etc. [9-11, 14-16].

As KAP1 is vital for many cellular processes, it can be studied in different angles for the better understanding of cellular process. The expression level of KAP1 varies in cell types, and it is very difficult to get an indicible expression in model cell lines. So, the establishment of stable cell line, where KAP1 is inducibly expressed, is necessary. In this study, we have constructed a KAP1 inducibly stable cell line, and then analyzed the regulation of KAP1 expression with DOX by Western blotting. Here, the vector pcDNA5/FRT/TO/KAP1 was constructed mainly because pcDNA5/FRT/TO is an inducible expression vector, which was designed by using the system with the Flp-In ${ }^{\mathrm{TM}} \mathrm{T}-\mathrm{REx}^{\mathrm{TM}}$. This vector contains a hybrid human cytomegalovirus/TetO2 promoter for high level DOX regulated expression of target gene in many mammalian cells $[1,2,6]$. Moreover, there is a multiple cloning site in this vector with unique restriction sites to facilitate cloning one copy of gene into FLP recombination target (FRT) site for FLP recombinase-mediated integration of the vector into Flp-In ${ }^{\mathrm{TMT}}$ $\mathrm{REx}^{\mathrm{TM}}$ host cell, which can be easily identified with hygromycin resistancy [7].

We have also modified the vector that has $2 \times$ FLAG tag at 3 ' prime of polyclone site. Once established the inducible cell line, we have treated the cells with DOX and detected the expression of target genes in established inducible cells with either FLAG antibody or specific antibody from target gene(s) [6]. DOX is usually used as inducer for the expression of target gene.

In molecular biological studies, desired expression of a gene/protein is often necessary. Some genes are artificially over-expressed and some genes are down-expressed by applying molecular technologies for the functional studies of these target genes/ proteins. In this study, we have successfully established a stable cell line with inducible expression of KAP1 gene/protein. This stable cell line with induced KAP1 expression might be very useful for KAP1 functional studies.

\section{ACKNOWLEDGEMENTS}

This work was supported by the Education Department Foundation of Sichuan Province (14ZA0148), Luzhou Medical College Foundation (2011-17) and Luzhou City Special Fund (2013LZLY-J10). This work was also supported in part by the National Natural Science Foundation of China (81172049, 30371493), Science and Technology Innovation Team of Colleges and Universities of Sichuan Province (13TD0032). 


\section{REFERENCES}

1. Andersson, S., Davis, D. L., Dahlbäck, H., Jörnvall, H., Russell, D. W. (1989) Cloning, structure, and expression of the mitochondrial cytochrome P-450 sterol 26-hydroxylase, a bile acid biosynthetic enzyme. J. Biol. Chem. 264, 8222-8229.

2. Boshart, M., Weber, F., Jahn, G., Dorsch-Häsler, K., Fleckenstein, B., Schaffner, W. (1985) A very strong enhancer is located upstream of an immediate early gene of human cytomegalovirus. Cell 41 521-530.

3. Fu, J. J. (2012) Short Protocols in Medical Molecular Biology. China Medical Science Press, Beijing.

4. Fu, J., Zhang, L., He, T., Xiao, X., Liu, X., Wang, L., Yang, L., Yang, M., Zhang, T., Chen, R., Xu, J. (2012) TWIST represses estrogen receptor-alpha expression by recruiting the NuRD protein complex in breast cancer cells. Int. J. Biol. Sci. 8, 522-532.

5. Fu, J., Qin, L., He, T., Hong, J., Wong, J., Liao, L., Xu, J. (2011) The TWIST/Mi2/NuRD protein complex and its essential role in cancer metastasis. Cell Res. 21, 275-289.

6. Fu, J. J., Zeng, W. M., Li, Y. H., Guo, M. R. (2008) Establishment and characterization of inducible cell line with stable expression of Spata3. Chin. J. Cell Biol. 30, 247-250.

7. Gritz, L., Davies, J. (1983) Plasmid-encoded hygromycin B resistance: the sequence of hygromycin B phosphotransferase gene and its expression in Escherichia coli and Saccharomyces cerevisiae. Gene $25,179-188$

8. Iyengar, S., Farnham, P. J. (2011) KAP1 protein: an enigmatic master regulator of the genome. J. Biol. Chem. 286, 26267-26276.

9. Iyengar, S., Ivanov, A. V., Jin, V. X., Rauscher, F. J. $3^{\text {rd }}$, Farnham, P. J. (2011) Functional analysis of KAP1 genomic recruitment. Mol. Cell Biol. 31, 1833-1847.

10. Jakobsson, J., Cordero, M. I., Bisaz, R., Groner, A. C., Busskamp, V., Bensadoun, J. C., Cammas, F., Losson, R., Mansuy, I. M., Sandi, C., Trono, D. (2008) KAP-1 mediated epigenetic expression in the forebrain modulates behavioral vulnerability to stress. Neuron $60,818-831$.

11. Liu, L., Zhao, E., Li, C., Huang, L., Xiao, L., Cheng, L., Huang, X., Song, Y., Xu, D. (2013) TRIM28, a new molecular marker predicting metastasis and survival in early-stage non-small cell lung cancer. Cancer Epidemiol. 37, 71-78.

12. Moosmann, P., Georgiev, O., Le Douarin, B., Bourquin, J. P., Schaffner, W. (1996) Transcriptional repression by RING finger protein TIF1 beta that interacts with the KRAB repressor domain of KOX1. Nucleic Acids Res. 24, 4859-4867.

13. Reymond, A., Meroni, G., Fantozzi, A., Merla, G., Cairo, S., Luzi, L., Riganelli, D., Zanaria, E., Messali, S., Cainarca, S., Guffanti, A., Minucci, S., Pelicci, P. G., Ballabio, A. (2001) The tripartite motif family identifies cell compartments. EMBO J. 20, 2140-2151.

14. Silva, F. P., Hamamoto, R., Furukawa, Y., Nakamura, Y. (2006) TIPUH1 encodes a novel KRAB zincfinger protein highly expressed in human hepatocellular carcinoma. Oncogene 25, 5063-5070.

15. Wang, C., Ivanov, A., Chen, L., Fredericks, W. J., Seto, E., Rauscher, F. J., Chen, J. (2005) MDM2 interaction with nuclear corepressor KAP1 contributes to p53 inactivation. EMBO J. 24, 3279-3290.

16. Yokoe, T., Toiyama, Y., Okugawa, Y., Tanaka, K., Ohi, M., Inoue, Y., Mohri, Y., Miki, C., Kusunoki, M. (2010) KAP1 is associated with peritoneal carcinomatosis in gastric cancer. Ann. Surg. Oncol. 17, $821-828$. 